\title{
A utilização de Interação Natural em Ambientes Tridimensionais para Treinamento no Setor Elétrico
}

\author{
Rafael H. A. de Castro ${ }^{1}$, Anna C. S. Medeiros ${ }^{1}$, Felipe G. dos Santos ${ }^{2}$, Tatiana A. \\ Tavares $^{1}$, Iguatemi E. da Fonseca ${ }^{1}$ \\ ${ }^{1}$ Centro de Informática \\ Universidade Federal da Paraíba (UFPB) - João Pessoa, PB - Brasil \\ ${ }^{2}$ Departamento de Informática \\ Universidade Federal de Campina Grande (UFCG) - Campina Grande, PB - Brasil \\ \{rafaelhenriquec, linnamedeiros\}@gmail.com, santos-fg@homail.com, \\ tatiana@lavid.ufpb.br, iguatemidci.ufpb.br
}

\begin{abstract}
Procedure Simulators have been used in several areas to help the execution of various tasks. The use of interfaces which facilitate the manipulation of the actions at different stages of execution of the task in the context of the simulator can be an important component to facilitate use by the professionals. The main objective of this study is to design and implement the integration of natural interaction, through gestures, into procedure simulators for the energy sector.
\end{abstract}

Resumo. Simuladores de procedimentos tem sido utilizados em diversas áreas para auxiliar a execução de diversas tarefas. $O$ uso de interfaces que facilitem a manipulação das ações, nas diversas fases da execução, da tarefa no contexto do simulador pode ser uma importante componente para facilitar o uso por partes dos profissionais. $O$ objetivo principal desse trabalho é projetar e executar a integração de interação natural, através de gestos, em simuladores de procedimentos para o setor elétrico.

\section{Introdução}

O uso de Realidade Virtual está cada vez mais presente, seja por conta do avanço tecnológico, que proporcionam computadores com maior desempenho, ou pela baixa do custo, que aumenta o acesso às tecnologias. Por isso é possível encontrar um maior número de novos estudos e pesquisas que envolvam modos de utilizar simuladores virtuais como meio de treinamento nas mais diversas áreas, como em (PONDER et al., 2003), (MENDES et al., 2010) e (VORA et al., 2002). O uso desses simuladores permite um treinamento auxiliar sem risco, de custo mais baixo e de fácil reprodução, podendo assim, atingir um maior número de pessoas sem a necessidade do trabalho extra da construção de um novo cenário físico de treinamento, e em casos de sistemas que não necessitem de dispositivos especiais, sem custo extra.

Buscando melhorar tais sistemas, é importante o estudo da melhor maneira de interagir com ele, é aí onde a Interação Natural pode ser útil, pois, como dito por Valli (2007), trata-se da busca de uma interação mais intuitiva procurando usar ações realizadas no dia-a-dia, como fala e gestos. Em (PANAIT et al., 2009) pode ser visto uma melhora no treinamento laparoscópico, em procedimentos mais complexos, com o uso de dispositivo háptico (retorno de força) no simulador. Os trabalhos (TAVARES et al., 2013), (CASTRO; MEDEIROS; TAVARES, 2012) e (MEDEIROS et al., 2012) 
mostram um exemplo da aplicação de gestos como alternativa para a interação em sistemas de telemedicina destinados ao ensino de estudantes de Medicina e Saúde em um hospital universitário.

O setor elétrico pode usufruir do uso de simuladores, visto que é repleto de operações complexas e perigosas, que proporcionam risco de morte e risco a patrimônio tangível e intangível. É importante, portanto, que os profissionais do setor sejam altamente qualificados, pois não são admitidos erros operacionais.

Como pode ser visto em (COGE, 2013) os acidentes fatais, ao longo dos anos, têm como causas principais: queda, origem elétrica e veículos. Tais causas podem ser evitadas, especialmente as duas primeiras, pois dependem exclusivamente do cumprimento de procedimentos técnicos de trabalho (planejamento da segurança no trabalho, observação das frentes de trabalho, procedimentos de trabalho escritos - o passo a passo, treinamento da força de trabalho, além do compromisso gerencial, etc), elementos constantes do SGTS - Sistema de Gestão do Trabalho Seguro. Tendo em mente o que foi mostrado acima, é importante o treinamento eficaz dos operadores da área elétrica. Para isso, esse trabalho procura melhorar a experiência com o simulador de procedimentos no setor elétrico ATreVEE 3D, apresentado em (SANTOS, 2013), com a adição de Interação Natural (IN), usando como meio de entrada comandos gestuais e o dispositivo Leap Motion (Leap Motion, 2013).

\section{Trabalhos Correlatos}

Cardoso et al. (2013) mostra o trabalho desenvolvido pela Faculdade de Engenharia Elétrica da UFU (Universidade Federal de Uberlândia) em parceria com a empresa de energia elétrica de Minas Gerais CEMIG, o sistema VRCEMIG. Esse sistema permite o controle de subestações pelo ambiente virtual, em tempo real, as mudanças no sistema afetam a subestação real e a mudança nos componentes da subestação atualiza o sistema em tempo real. Com isso procura-se proporcionar um ambiente que permite controle das diversas subestações da empresa, como um sistema que permite o treinamento de operadores em ambiente seguro e de baixo risco. $\mathrm{O}$ trabalho apresenta também pesquisas realizadas pelo Grupo de Realidade Virtual e Aumentada da UFU com sensores EMG ou reconhecimento de gestos juntamente com realidade aumentada.

O trabalho apresentado em (BELLOC, 2011) propõe um arcabouço para auxiliar o desenvolvimento de simuladores de procedimento com RV e para validar tal proposta são utilizados dois casos de uso na área elétrica. $\mathrm{O}$ primeiro sendo um simulador para a troca de cruzeta de um poste em linha viva, procedimento esse demorado e de alto risco, por isso a importância de um bom treinamento. A aplicação utiliza como meio de interação o mouse e as ações são escolhidas através de uma lista de opções mostradas ao selecionar um objeto interativo no cenário. O segundo sistema desenvolvido, realizado com o objetivo de enriquecer o material utilizado no treinamento, trata-se de procedimentos de montagem e desmontagem de uma Unidade Geradora, através de execução meramente ilustrativa (com a manipulação somente das principais peças da $\mathrm{UG})$.

Barata (2010) apresenta uma solução de software para a criação de simulações em RV de processos operacionais industriais (um sistema autoral), que permite a criação de simulações sem conhecimento de programação. Para validar o sistema foi 
desenvolvido um estudo de caso na área do setor elétrico, onde é simulada a partida de uma Unidade Hidrogeradora (UHG). O processo de partida na UHG pode ser monitorado (na Usina Hidrelétrica de Tucuruí) através de um computador, coletando e enviando comandos para a UHG através de sensores e relés instalados por todos os equipamentos da unidade. $O$ simulador apresenta a interface do computador de monitoração, onde o usuário pode utilizar a interface igual a real. Apresentando animações do que ocorre nos equipamentos no decorrer do processo.

Em (PAMPLONA JR.; RIBEIRO FILHO; MOREIRA, 2010) é apresentado mais um caso de uso do sistema de autoria mostrado em (BARATA, 2010), dessa vez, o cenário é a manutenção da válvula atuadora do regulador de velocidade do prédistribuidor da turbina. A válvula atuadora é um componente que transforma um sinal elétrico de pequena potência, emitido pelo controlador de velocidade em um sinal hidráulico capa de movimentar a válvula de controle do servomotor principal responsável pela movimentação das pás do rotor e do pré-distribuidor.

Sousa et al. (2008) apresenta um ambiente de treinamento de manutenção em uma Unidade Hidrelétrica de Energia (UHE), o trabalho mostra dois módulos, um módulo de montagem e um de manutenção. O módulo de montagem permite o treinando executar virtualmente os procedimentos de montagem do concreto e de peças da UHE. O segundo módulo (de manutenção) permite o treinamento de procedimentos de manutenção, realizando operações de desmontagem e montagem de peças e equipamentos da UHE, baseado nos manuais de operação e manutenção da turbina fornecidos pela ELETRONORTE.

Arendarski, Termath e Mecking (2008) apresentam uma plataforma de visualização interativa para desenvolvimento e treinamento virtual, sistema esse usado para criar cenários animados 3D para diversas funções e áreas. Em particular é mostrado um cenário virtual que apresenta um transformador de energia de voltagem ultra- alta tensão. O cenário descrito trata-se da preparação, desmontagem e desconexão de um transformador para que ele seja transportado. O simulador inclui passos preparatórios, como isolar a área de trabalho e preparar os equipamentos que serão utilizados.

Em (GARANT et al., 1995) é apresentado o ESOPE-VR, um sistema de RV utilizado para o treinamento de operários que trabalham em comutação de energia elétrica ou estações de distribuição, permitindo os operários a praticarem rotinas que teriam que realizar no curso do seu trabalho. O maior foco da interação manual no simulador está nos painéis de controle nos quais os equipamentos podem ser operados, para interagir com os botões e alavancas do painel é utilizado um modelo de mão 3D controlado por um dispositivo de 6 graus de liberdade (6-DoF: 6-Degree-of-Freedom, que permite o movimento nos eixos $\mathrm{x}, \mathrm{y}, \mathrm{z}$ e a rotação em cada um dos eixos). $\mathrm{O}$ sistema ainda permite o uso de comandos de voz para contralar a direção de movimento e disponibiliza como retorno imagem tridimensional (estereoscópica) e avisos de áudio.

(LOS ARCOS et al., 1999) apresenta um sistema para o treinamento de operadores do setor elétrico constituído de dois sistemas independentes: o Simulador SGI (Sistema Gráfico Interativo), utilizado em treinamento e reciclagem dos processos dos operadores em ações nas subestações onde não há automação ou instalações para controle remoto; e o Assistente utilizado para auxiliar o usuário no processo de treinamento, provendo assistência quando o usuário erra ou quando requisitado. 
O trabalho apresentado em (BURIOL, 2011) mostra uma plataforma desenvolvida na UFPR (Universidade Federal do Paraná) de treinamento no setor elétrico. O sistema possibilita o treinamento da troca de cruzeta de postes elétricos em linha viva, para isso o sistema utiliza dois Wii Remotes (Controle do console Nitendo Wii, que possui sensor infravermelho), duas TVs estereoscópicas e óculos para visualização 3D, LEDs infravermelhos para referências, e dispõe também do uso de uma cesta isolada real, dentro da qual o eletricista permanece para a manutenção dos postes reais.

Os Wii Remotes são utilizados como apontador e para realizar o rastreio de cabeça. Para manipular interagir com a cena, o usuário utiliza um Wii Remote para apontar para a posição que deseja, além de poder rotacionar e mover a câmera para mudança da área de visualização da cena, isso é feito utilizando LEDs infravermelhas presas no friso do televisor, que são captadas pela câmera infravermelha do Wii Remote e partir da posição do controle em relação as LEDs são calculadas as coordenadas do cursor no cenário. Para o rastreio de cabeça um par de LEDs são colocados no óculos para visualização 3D, e um Wii Remote é posicionado em cima das TVs com a câmera infravermelha voltada para o usuário, com as coordenadas dos dois LEDs o sistema rastreia a posição e rotação da cabeça e utiliza esses dados para movimentar a câmera para reproduzir uma visualização que respondam a movimentos feitos pelo usuário. $\mathrm{O}$ simulador permite com o uso do controle a manipulação dos objetos de cena, apontando o objeto desejado e pressionando um botão do controle para "pegá-lo". O treinamento é realizado com o treinando dentro da cesta isoladora e utilizando os EPIs (Equipamentos de Proteção Individual) para reproduzir as condições que o usuário estaria na situação real. A Tabela 1 lista os trabalhos supracitados destacando as tecnologias e dispositivos de interação utilizados.

Tabela 1 - Trabalhos Correlatos.

\begin{tabular}{|c|c|c|}
\hline Trabalho & Tecnologia & Dispositivos \\
\hline (CARDOSO et al., 2013) & RV/ Estereoscopia & $\begin{array}{l}\text { Mouse/ Teclado/ Joystick/ } \\
\text { Gamepad/ Óculos }\end{array}$ \\
\hline (BURIOL, 2011) & $\begin{array}{l}\text { RV/ Estereoscopia/ Rastreio de } \\
\text { Cabeça/ Ray Casting }\end{array}$ & $\begin{array}{l}\text { Wii Remotes/ TVs } \\
\text { Estereoscópicas }\end{array}$ \\
\hline (BELLOC, 2011) & RV & Mouse \\
\hline (BARATA, 2010) & RV & Mouse/ Teclado \\
\hline $\begin{array}{l}\text { (PAMPLONA JR.; RIBEIRO } \\
\text { FILHO; MOREIRA, 2010) }\end{array}$ & RV & Mouse/ Teclado \\
\hline (SOUSA et al., 2008) & RV & Mouse/ Teclado \\
\hline $\begin{array}{c}\text { (ARENDARSKI; TERMATH; } \\
\text { MECKING, 2008) }\end{array}$ & RV & Mouse/ Teclado \\
\hline (LOS ARCOS et al., 1999) & RV & Mouse \\
\hline (GARANT et al., 1995) & $\begin{array}{c}\text { RV/ Estereoscopia/ } \\
\text { Reconhecimento de Fala }\end{array}$ & $\begin{array}{l}\text { 6-DoF Mouse/ Shutter Glasses/ } \\
\text { Microfone }\end{array}$ \\
\hline
\end{tabular}

\section{A Interação Natural no Ambiente ATreVEE 3D}

\subsection{ATreVEE 3D}

O simulador de procedimentos no setor elétrico ATreVEE 3D pretende auxiliar o treinamento de profissionais do setor elétrico, tendo em vista o alto risco das atividades por eles realizadas. Esses profissionais precisam ser altamente qualificados, e essa ferramenta propõe ajudar em tal qualificação, permitindo também a realização da 
simulação a qualquer momento para que o operador possa rever os conceitos e instruções para a realização do procedimento quando necessário. Com isso visa-se ajudar os profissionais a trabalharem com mais segurança além de diminuir a ocorrência de problemas na rede elétrica, o que pode afetar diversas pessoas.

Durante o longo percurso da energia elétrica entre as usinas e os centros consumidores nas redes de distribuição existem elementos importantes das redes de transmissão que são os isoladores de vidro ou porcelana, que sustentam os cabos e impedem descargas elétricas durante o trajeto. Como pode ser visto em (VILELA, 2002) citado em (SANTOS, 2013), as falhas em isolamentos podem causar, desde interferências em sinais de rádio ou televisão, até curtos-circuitos que podem retirar a linha ou subestação de operação. A interrupção temporária de suprimento acarreta prejuízos às unidades consumidoras e à concessionária de energia. Para a concessionária, falhas em isolamentos podem resultar em danos materiais próprios e de terceiros, custos de manutenção, perda de faturamento e, em muitos casos, multas impostas por parte das agências reguladoras.

O simulador ATreVEE 3D busca evitar esses problemas com o treinamento do procedimento de substituição de isoladores em linha ativa e desativa em postes trifásicos com tensão de $13,8 \mathrm{kV}$. O simulador é composto por dois cenários, o cenário de treinamento principal e um cenário de preparação onde são escolhidos os equipamentos necessários. O cenário de preparação possui maior liberdade de interação, nele é controlado um avatar, para representar a chegada à empresa e depois é necessária a escolha dos equipamentos necessários para a substituição do isolador.A escolha é feita em meio a alguns objetos disponíveis em uma sala, controlando o braço do operador virtual com o mouse e teclado para pegar os objetos desejados. No cenário principal, execução da troca de isoladores, a interação é realizada por meios de botões na interface gráfica que representam as ações, como pode ser visto na Figura 1 . O procedimento é realizado através da escolha da ação que se deseja realizar, por meio do botão correspondente, onde a ação é mostrada por meio de animação.

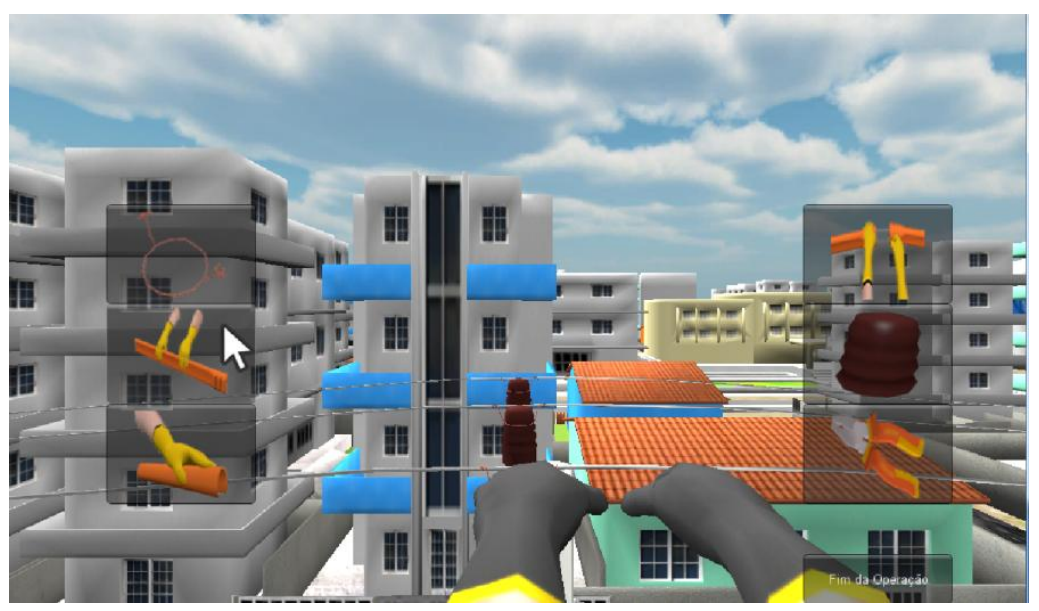

Figura 1 - Escolha da Ação com o Cursor.

\subsection{Suporte a IN}

Para a adição de IN ao simulador a arquitetura foi alterada de modo que futuramente o simulador não sofra modificação ao serem adicionados novos dispositivos. A Figura 2 mostra a arquitetura, na qual o módulo Interface do Usuário 
tem a função de fazer o tratamento dos dados brutos recebidos do dispositivo (no caso, o controle Leap Motion) traduzindo-os em comandos para a interface, assim, pode-se adicionar suporte para novos dispositivos com a adição de novos módulos para tratar seus dados e traduzi-los para os comandos da interface.

No piloto, os dados recebidos do controle Leap Motion são tratados com o uso do Leap Motion SDK e então utilizados para o controle dos avatares das mãos e da execução da ação "fechar mão" que é utilizada no simulador para pegar as ferramentas que estão disponibilizadas em cena. Os dados do rastreio são acessados a partir dos comandos do SDK, adquirindo posição das mãos e dos dedos rastreados, a partir dessas informações são definidas a posição das mãos dentro do cenário.

O controle Leap Motion foi escolhido no uso desse trabalho, por prometer uma precisão alta, permitindo o uso de comandos gestuais das mãos mais complexos, O que é importante para o procedimento que está sendo trabalhado, onde o trabalho é realizado por gestos das mãos, e não do resto do corpo. Foi pensado também no uso do Microsoft Kinect, mas acredita-se que não se encaixa no cenário proposto, necessitando testes para comprovar a inviabilidade.

\subsection{Cenários Desenvolvidos com IN}

A aplicação piloto tem como meio de entrada o controle Leap Motion, por meio do qual é feita a interação com o cenário virtual utilizando os dados fornecidos do rastreio das mãos. Como pode ser visto na Figura 4, o aplicativo possui dois modelos 3D que representam as mãos rastreadas e com esses modelos é possível saber com que parte do cenário está sendo realizada a iteração.

A atividade a ser realizada nesse cenário piloto, como foi dito anteriormente, é a escolha dos equipamentos que são necessários para a manutenção dos postes. Para pegá-los no cenário virtual, deve-se tocá-los, usando o modelo como referência de onde se encontra a mão rastreada dentro do cenário virtual, e fechar a mão. Após pegar todos os itens necessários para a realização da operação, a cena é finalizada. Pode ser que o usuário simplesmente esqueça de apanhar uma ferramenta ou tente pegar uma ferramenta que não é necessária na operação. Ao término da cena, o ATreVEE 3D fornece um relatório informando quais foram os erros e acertos, contribuindo dessa forma com o treinamento do profissional para a realização dessa operação. 


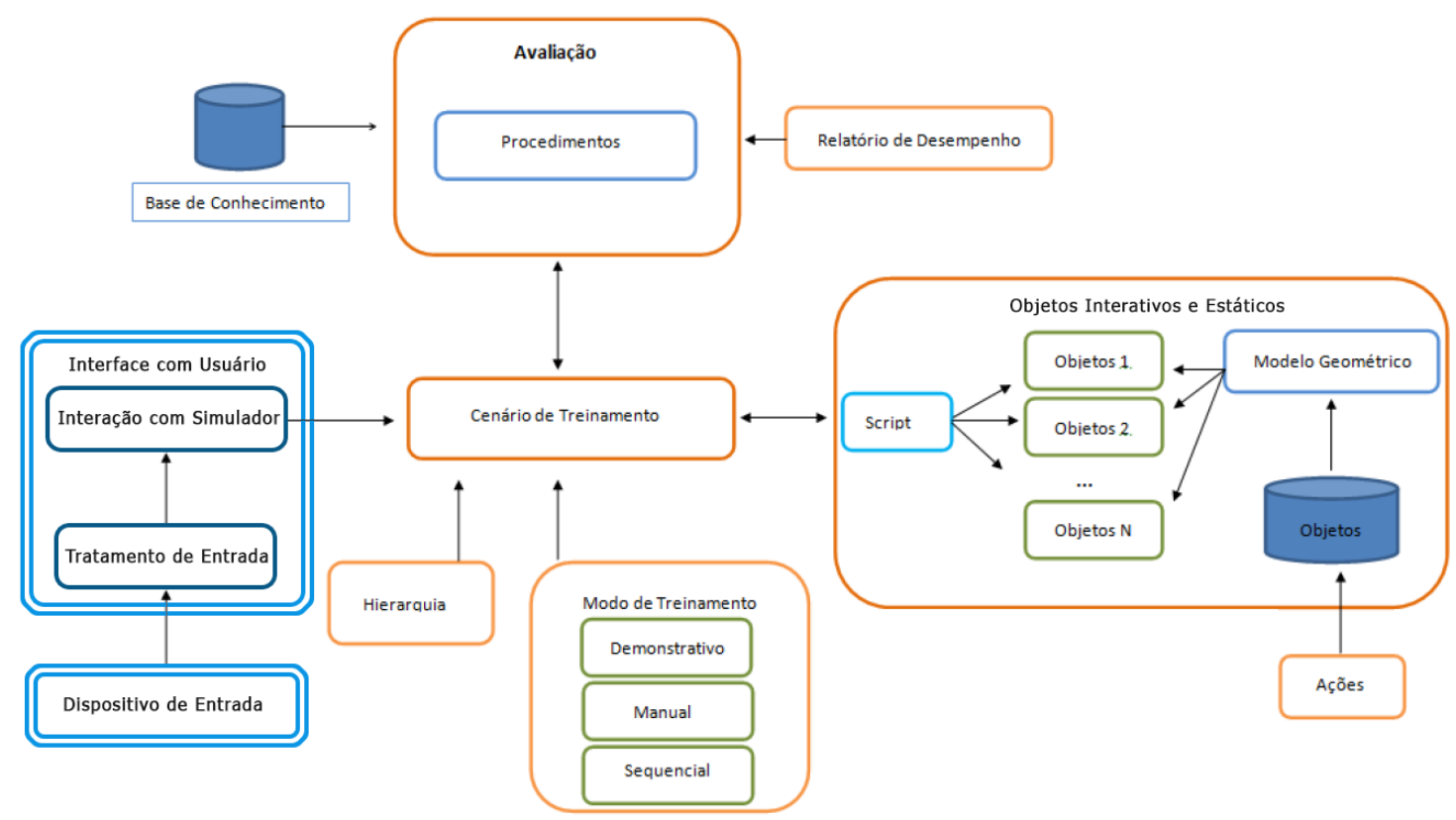

Figura 2 - Arquitetura

A navegação pelo cenário é realizada totalmente a partir do rastreio da mão, para locomover o "jogador", e com isso as mãos e a câmera, para a frente basta mover uma mão ou as duas em direção ao monitor, enquanto que para mover para trás deve-se mover a(s) mão(s) na direção contrária. Para evitar que todo movimento mova também o personagem e não só as mãos dos cenários foram atribuídos limiares que determinam a distância do dispositivo para que o movimento do personagem seja executado, caso esses limiares não sejam ultrapassados o rastreio das mãos movimentará apenas as mãos virtuais e os objetos com os quais elas estão interagindo.

É possível também a rotação do personagem, tanto horizontalmente como verticalmente, sendo que, na direção vertical, o ângulo de rotação é limitado, para permitir apenas uma melhor visualização quando necessário. De modo semelhante ao movimento a rotação é realizada com limiares nas coordenadas $\mathrm{X}$ e $\mathrm{Y}$ do rastreio, enquanto o movimento utiliza da coordenada Z. Na Figura 3 (a) pode-se ver um esquema de como é feito a navegação como descrita acima, onde é definido uma área dentro do alcance do controle Leap Motion e ao ultrapassá-la é iniciado o controle do personagem em vez do controle das mãos no cenário. Já na Figura 3 (b) temos a representação do gesto realizado para pegar os equipamentos na cena.

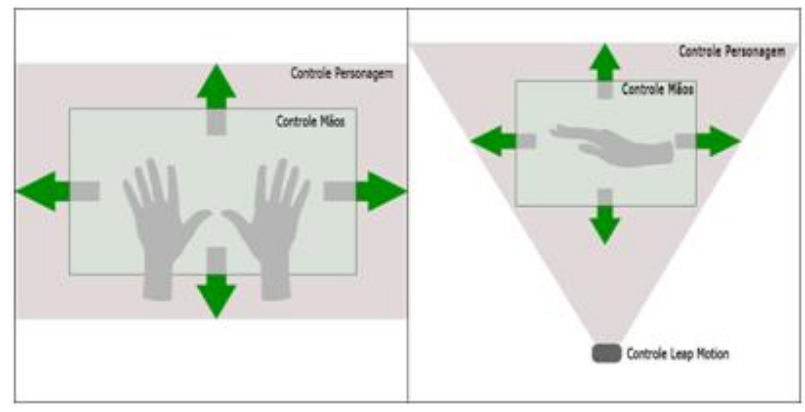

(a)

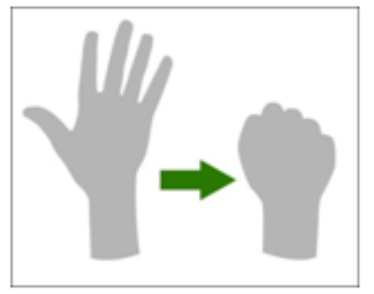

(b)

Figura 3 - (a) Controle Mãos e Personagem. (b) Gesto para "Pegar" 
O resultado final do gesto para "pegar" (Figura 3 (a)) pode ser visualizado na Figura 4. Nesse cenário o operador deve escolher os equipamentos que serão necessários para a realização da manutenção que estão em uma sala dispostos em armários e bancadas. Para tanto, o usuário deve utilizar o gesto "pegar" para manipular os itens corretos.

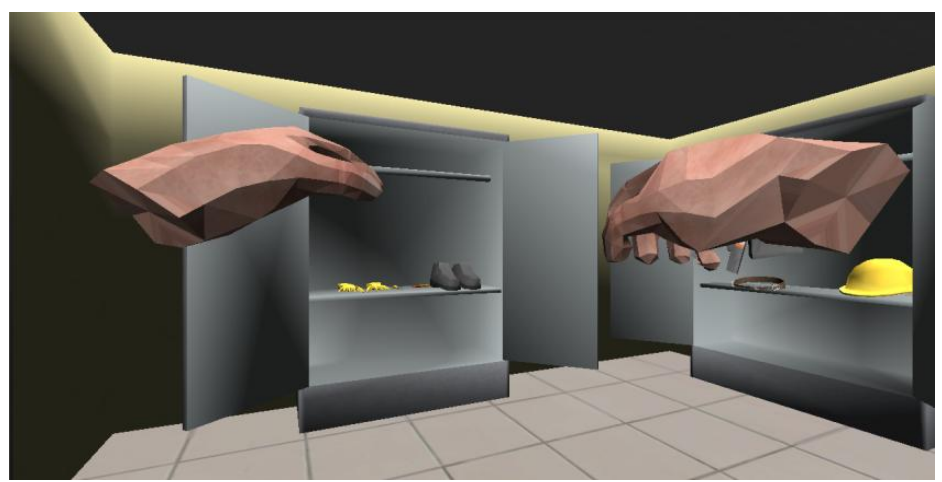

Figura 4 - Aplicação Piloto

\subsection{Testes Iniciais}

Para validar a funcionalidade da nova interface natural integrada ao ATreVEE 3D, foram feitos testes iniciais com voluntários da área de computação (Figura 5), sendo eles alunos de graduação e pós-graduação pesquisadores do laboratório LAVID (Laboratório de Aplicações de Vídeo Digital). Esse teste inicial nos ajudou a compreender melhor o uso do dispositivo Leap Motion, bem como, sua integração ao ATreVEE 3D. O passo seguinte será envolver um número maior de voluntários para qualificar o cenário comparando-o com o modo de interação via mouse e teclado. Nossa interação é observar como o uso do Leap Motion e da IN pode afetar a percepção do usuário e sua relação de envolvimento com o sistema.No intuito de obter melhores resultados em testes futuros procura-se melhor definir os gestos utilizados em outros cenários dessa aplicação. Outra ponto importante será o feedback de representantes do público-alvo nos próximos testes. Para tanto, pretendemos envolver funcionários do setor elétrico, os quais realizam essas funções no seu trabalho diário.

\section{Conclusão}

Esse trabalho procura enriquecer a experiência para o treinamento no setor elétrico, buscando aprimorar o simulador ATreVEE 3D com a adição de interação gestual na realização do treinamento do procedimento, troca de isoladores.

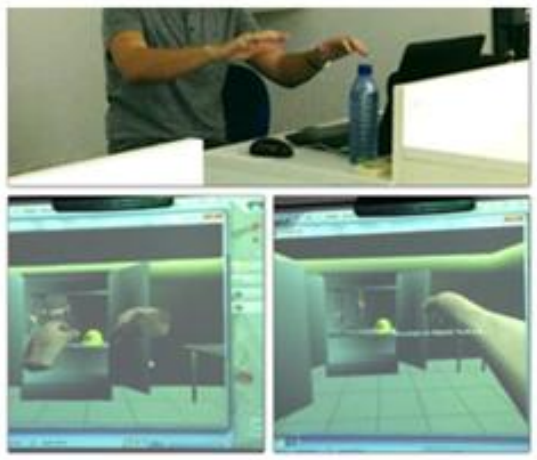

Figura 5 - Testes com Usuários 
Alguns desafios espera-se encontrar durante o desenvolvimento dessa integração, como a redefinição de todo o cenário, pois o cenário é construído de forma que toda interação é realizada totalmente por meio de botões que ativam animações que demonstram a ação escolhida, sendo então necessário transformar essas animações em ações realizadas pelo usuário com o intuito de melhorar a experiência do treinamento. Além disso é importante buscar os melhores comandos a serem utilizados para a interface, sendo assim um estudo e planejamento deve ser feito tanto para decidir gestos que serão utilizados na interação com o cenário, como definir como serão realizados comandos operacionais do simulador, se serão realizados via o dispositivo ou por meios tradicionais de entrada. Outro ponto que se deve ter em mente é a possibilidade de ser necessário implementar um módulo de reconhecimento de gestos, caso a complexidade dos movimentos desejados para uma interação intuitiva sejam além dos gestos prédefinidos disponibilizados pelo SDK. Ao finalizar a adição da interação gestual em todo o cenário, será realizado testes com profissionais do setor elétrico com as duas versões do simulador e a aplicação de questionários, buscando verificar as vantagens e desvantagens da adição do novo dispositivo de interação.

\section{Referências}

ARENDARSKI, B.; TERMATH, W.; MECKING, P. Maintenance of Complex Machines in Electric Power Systems Using Virtual Reality Techniques, 2008.

BARATA, P. A. B. Projetos e Implementação de um Sistema de Autoria para Animações, Simulações e Treinamentos em Realidade Virtual. Dissertação de Mestrado. Programa de Pós-Graduação em Engenharia Elétrica da Universidade do Pará, Belém-PA, Setembro 2010.

BELLOC, O. R. Um Arcabouço para o Desenvolvimento de Simuladores de Procedimentos. Dissertação de Mestrado. Escola Politécnica da Universidade de São Paulo, 2011.

BURIOL, T. M. Convergência de Games e Realidade Virtual para Treinamento de Manutenção em Redes de Energia em Linha Viva. Defesa de Doutorado. Programa de Pós-Graduação em Métodos Numéricos em Engenharia. Universidade Federal do Paraná, Curitiba, 2011.

CARDOSO, A. et al. VRCEMIG: a Virtual Reality System for Real Time Control of Electric Substations. IEEE Virtual Reality 2013, Orlando - FL. EUA, 2013. 165166.

CASTRO, R. H. A. D.; MEDEIROS, A. C. S.; TAVARES, T. A. Natural Interaction for 3D Manipulation in Telemedicine: A Study Case developed for Arthron Video Collaboration Tool. Workshop de Realidade Virtual e Aumentada - WRVA 2012, Paranavaí, 2012.

COGE. Relatório 2012 - Estatísticas de Acidentes do Setor Elétrico Brasileiro. Fundação COGE, 2013. Disponivel em: <http://www.funcoge.org.br/csst//relat2012/index.html>. Acesso em: Fevereiro 2014.

GARANT, E. et al. A Virtual Reality Training System for Power-Utility Personnel, 1995. 
LEAP Motion. Leap Motion, 2013. Disponivel em: <www.leapmotion.com/>. Acesso em: Dezembro 2013.

LOS ARCOS, J. L. et al. PDA: A Training Application to Electrical Substation Operation, 1999.

MEDEIROS, A. C. S. et al. Interação natural como apoio a atividade de telementoria em Telemedicina - WAIEd 2012. Workshop Ambientes Imersivos para Educação, Rio de Janeiro, 10, 2012.

MENDES, J. B. et al. Low Cost Helicopter Training Simulator: A Case Study from the Brazilian Military Police, 2010.

PAMPLONA JR., A.; RIBEIRO FILHO, M.; MOREIRA, C. Avaliação do Sistema de Autoria de Instruções Técnicas Virtuais, 2010.

PANAIT, L. et al. The Role of Haptic Feedback in Laparoscopic Simulation Training. Journal of Surgical Research 156, p. 312-316, 2009.

PANAIT, L. et al. The Role of Haptic Feedback in Laparoscopic Simulation Training. Journal of Surgical Research 156, p. 312-316, 2009.

PONDER, M. et al. Immersive VR Decision Training: Telling Interactive Stories Featuring Advanced Virtual Human Simulation Technologies. International Immersive Projection Technologies Workshop, 2003.

SANTOS, F. G. Plataforma Computacional para Ensino e Treinamento de Profissionais do Setor Elétrico. Dissertação de Mestrado. Programa de Pós-Graduação em Sistemas de Comunicação e Automação na Universidade Federal RURAL do Semi-Árido, Mossoró - RN, 2013.

SOUSA, M. P. A. et al. Maintenance and Assembly Training in a Hydroelectric Unit of Energy Using Virtual Reality Desktop. IEEE Latin America Transactions, Vol. 6, No. 5, Setembro 2008. 484-491.

TAVARES, T. A. et al. The use of natural interaction to enrich the user experience in telemedicine systems. International Conference on Human-Computer Interaction, HCI International 2013 - Posters Extended Abstracts. Las Vegas: [s.n.]. 2013. p. 220224.

VALLI, A. Notes on Natural Interaction. [S.1.]: [s.n.], 2007.

VILELA, T. F. Estimação Inteligente da Poluição de Isolamentos Elétricos Baseada nos Vecse Do Ruído Ultrassônico. Tese (Doutorado). Pós-Graduação em Engenharia Elétrica da Universidade Federal de Campina Grande, Campina Grande - PB, 2002.

VORA, J. et al. Using Virtual Reality Technology for Aircraft Visual Inspection Training: Presence and Comparison Studies. Applied Ergonomics 33, p. 559-570, 2002 . 